\title{
AL MARGINE DEI MARGINI: RIBELLIONE, ESPERIENZA DEL DOLORE E DENUNCIA SOCIALE IN LETTERIA MONTORO, DONNA SICILIANA E SCRITTRICE DEL ROMANTICISMO
}

\author{
IN MARGIN OF THE MARGINS: RIBBONATION, EXPERIENCE OF DOLOR AND \\ SOCIAL DOCUMENT IN MONTORO LETTER, SICILIAN WOMAN AND SCRIT- \\ TER OF ROMANTICISM
}

Daniela Bombara

Università di Messina

\section{Riasunto:}

Questo lavoro esamina la produzione della messinese Letteria Montoro (1825-1893), poetessa e autrice di Maria Landini, romanzo popolare con protagonista una donna ribelle, che combatte per affermare la propria libertà nello squallido scenario di una società degradata. Volontà di impegno politico, critica sociale e 'cattiveria rappresentativa' configurano la produzione di Montoro, apparentemente marginale e del tutto ignorata dalla critica, come perfettamente inserita nel contesto ideologico del Romanticismo italiano.

\section{Parole chiave:}

Messina, romanzo storico, donna ribelle, critica sociale, studi di genere, Romanticismo

\section{Abstract:}

This essay intends to examine the literary works of Letteria Montoro (Messina 18251893), poet and novelist. She writes Maria Landini, a historical - contemporary novel, where the main character is a rebel woman who fights to preserve her freedom of choice in the moral squalor of a corrupted society. Letteria Montoro is currently unknown and her works are surely marginalized in the criticallandscape, but they are very interesting as they show her willingness to describe the world of the time with social involvement and realism, in agreement with the Romantic ideology

\section{KEY WORD:}

Messina, historical novel, rebel woman, social criticism, gender studies, Romanticism. 
In una storia di marginalità quale quella di Letteria Montoro, autrice siciliana ogg del tutto dimenticata, vissuta in un contesto culturale periferico, e ulteriormente isolata dal suo essere donna e scrittrice in una società a dominanza maschile, ci sembra opportuno iniziare dall'estremo limite della sua esistenza: l'epitaffio ci fornirà dunque il quadro di riferimento essenziale per iniziare il nostro discorso:

Qui per volere del Comune/ l'ala dell'oblio non graverà sulle ceneri/ di LETTERIA $\mathrm{MONTORO/che} \mathrm{l'anima} \mathrm{forte} \mathrm{ed} \mathrm{eletta/} \mathrm{trasfuse} \mathrm{in} \mathrm{versi} \mathrm{soavii} \mathrm{ed} \mathrm{in} \mathrm{prose} \mathrm{eleganti/}$ donna di spiriti liberali/ confortò i fratelli che combattevano/ per la redenzione d'Italia/ li seguì nell'esilio/ e ad essi tornati in patria/ sacrificò cristianamente la vita/ mirabile esempio di fraterno affetto!/ 19 aprile 1825 - 1 agosto 1893 (Attard 1991: 34).

Siamo nel cimitero di Messina; la lapide è posta nella cella sotterranea della Galleria monumentale, ma il terremoto del 1908 distrugge cose e persone, spazzando via la memoria della città; il ricordo della scrittrice scompare quindi insieme ai suoi resti umani. È degno di nota come queste poche, ma dense frasi, costituiscano la traccia più significativa per percorrere a ritroso le tappe di un'esistenza e presenza letteraria della quale abbiamo minime testimonianze. Ci resta infatti un solo, sintetico profilo biografico, redatto dallo storico messinese Gaetano Oliva; egli include Letteria Montoro come unica donna nel novero dei Messinesi illustri:

Nata il 19 aprile 1825, morta il primo agosto 1893, fu donna di altissima intelligenza e di cuore immensamente benefico e generoso. Ella amò il sacrificio sino all'eroismo e benché fornita di singolare bellezza nel volto e nella gentil persona, compresse ogni sentimento dell'animo tenero e poetico, e rifiutò i vari matrimonii che le si offrirono per rimanere in famiglia, la quale avea indispensabile bisogno di lei. Poetessa nata, scrittrice forbita e gentile, di quando in quando, se le cure domestiche glielo consentivano, affidava i suoi sentimenti alla carta, e scrisse parecchie poesie qualche romanzo senza pretesa di gloria; ma ciò malgrado molte suechie poesie e qua ebbero pubblicita, ed ell bormo soprattutto la lettura del Leopardi, la cui patetica poesia prediligeva (Oliva 1954 292- 293)

Vocazione artistica e sacrificio di sé; il discorso di Oliva si muove fra questi due poli, evitando però di fare accenno alla dimensione politica dell'estrema 'generosità' di Letteria, confinando quindi il personaggio nel consueto ruolo femminile materno/ assistenziale. ${ }^{1}$ Dobbiamo integrare con dati interni ai testi: Montoro è figlia di un esule, come apprendiamo dalla prefazione al suo unico romanzo edito, Maria Landini, in cui afferma di aver ritratto nella figura del proscritto Antonio il proprio padre; la ragazza

L'epitaffio sottolinea invece con forza, come si è visto, l'impegno politico di Letteria; un dato confermato dalle parole di Gaetano La Corte Cailler, storico siciliano: "Poetessa e scrittrice erudita, autrice di vari lavori letterari, esule per la redenzione della patria, è morta nel 1893 a 68 anni in Messina". (La Corte Cailler 1914: 23) quindi conduce la sua giovinezza nel segno dell'esclusione, ma insieme delle idealità democratiche, e sappiamo che partecipa alla rivoluzione del 1848 collaborando a giornale patriottico "L'Aquila Siciliana", nato il 16 febbraio, a ridosso dei moti (Tomeucci 1963: 121, 147). L'esilio appare a questo punto una logica conseguenza; conosciamo troppo poco le vicende biografiche di Montoro per comprendere l'effettiva dinamica di un evento che, per quanto inusuale quando la protagonista è donna, è testimoniato nei documenti d'epoca in riferimento a figure di mogli e madri: sembra infatti che la donna esule rivestisse un ruolo di mediazione fra confinati politici e strutture di governo (Tafuro 2011: 56)

Il successivo rientro a Messina comporta un ritorno alla 'normalità' e l'assunzione di un ruolo defilato? Anche in questo caso l'esame delle opere integra un quadro biografico molto incerto: nel 1886 un'ormai anziana Letteria scrive un prolisso componimento in morte del fratello, il sacerdote Francesco Montoro, affermando d essersi dovuta occupare in prima persona della famiglia d'origine per consentire al più anziano parente di svolgere diversi e onerosi incarichi pubblici ( Montoro 1886) Il testo poetico traccia il quadro rassicurante di una donna di grande abnegazione che accetta di vivere, dopo il glorioso periodo rivoluzionario, all'ombra del fratello, più noto in città: direttore del Collegio Peloritano messinese, e direttore spirituale del liceo ginnasio Maurolico, dedito ad iniziative filantropiche e ad una meritoria attività in campo educativo ed assistenziale.

Ma è proprio dagli anni '50, in realtà, che data la produzione letteraria di Montoro; e si tratta di un'attività diversificata, che spazia dalla prosa - novelle e romanzi - alla poesia: lirica, d'occasione, civile, patriottica. Sono passati due anni dal '48, quindi da un'esperienza politica che costituisce uno spartiacque nella società siciliana determinando il consolidarsi delle idealità risorgimentali all'interno della nascente classe borghese, e comportando l'assunzione di un ruolo autonomo da parte di donne che "scrivono e leggono, inventano e speculano senza vedere la cultura femminile solo al servizio del ruolo familiare" (Fiume 2006: 745). Questa esperienza ha costituito sicuramente un forte momento identitario anche per Letteria, che vi ha partecipato con convinzione; ma ora, una volta che la fase della partecipazione attiva è conclusa, la giovane donna sceglie la scrittura per continuare a far sentire la sua voce, piuttosto che annullarsi silenziosamente in quelle occupazioni di economia domestica così pervasive da costituire "croce e delizia, regno e prigione delle donne dell'Ottocento" (Fiume 2006: 745). La biografia di questa semisconosciuta letterata è quindi per vari aspetti sorprendente: esclusi dal suo orizzonte mentale marito e figli, Montoro manifesta una volontà di azione politica che sfocia in un esilio sofferto; il ritorno a Messina negl anni '50 comporta uno slittamento di interesse dall'ambito della società reale, in cui 
la rivoluzione è ormai inattuabile, al mondo virtuale della letteratura, dove ancora è possibile l'impegno.

Letteria Montoro ci appare quindi una figura di sicuro interesse, ma dimenticata dalla critica: se escludiamo i repertori che si occupano di donne notevoli, dove sono presenti alcune sparse informazioni, ${ }^{2}$ troviamo in opere di diverso genere telegrafici accenni alla scrittrice riferiti ad aspetti parziali della sua produzione; ${ }^{3}$ in anni recent Rita Verdirame le dedica un trafiletto e una lunga nota nel suo Narratrici elettrici (18501950): nota poco dettagliata, in ogni caso, poiché Verdirame dichiara sostanzialmente di ignorare quest'autrice:

La semisconosciuta rimatrice e romanziera messinese Letteria Montoro (1825 ?) redasse un'ode accorata Sulla tomba della chiarissima Mariannina Coffa poetessa netina [...] L'ode vide la luce per i tipi di Virzi nel 1878; dell'autrice si conservano pochissime notizie, probabilmente a motivo di una produzione povera e a diffusione eminentemente locale, s'ignora la data di morte e ogni altro dettaglio biografico. L'erudito Giovanni di Pietro, nel suo regesto Illustrazione di Scrittori biografico. Le icitimi 1830 a dittor

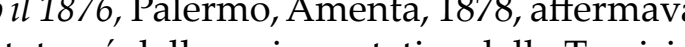
( Colonna né del vigore della Muzio Salvo, implicitamente testimoniando che s'era individuato in quegli anni un canone femminile regionale di riferimento, sebbene poi esso si sia rivelato di effimera durata. La Montoro doveva essere nota anche alle colleghe palermitane incontrate sulla ben confezionata testata cattolico- moderata La Donna e la famiglia, in vita tra il 1862 e il 1864 (dove scrissero anche Rosina Muzio Salvo, Mariannina Coffa Caruso e Concettina Ramondetta Fileti), le qual ebbero sicuramente agio di leggere il suo primo romanzo, Maria Landini, edito per l'appunto a Palermo, Clamis e Roberti, 1850, menzionato dal Cattaneo, "Sul Romanzo delle donne contemporanee in Italia". (Verdirame 2009: 59, 59 n. 125).

La menzione di Carlo Cattaneo potrebbe essere interessante, ma in realtà non ci dice nulla di sostanziale su Montoro: il critico infatti, dopo aver riassunto un romanzo

2 Ad esempio Oscar Greco nella sua Biobibliografia femminile italiana del XIX secolo non fornisce notizie biografiche sulla scrittrice, ma solo minime informazioni relative ad opere di Montoro di cui non si ha notizia da altre fonti: un Frammento di romanzo inedito, pubblicato nella Strenna venezian del 1866, e Ad un giacinto quartine, ne "La Missione delle donne", periodico diretto da Olimpia Saccati (Greco 1875: 341-2); telegrafico il profilo di Leone Carpi nel lavoro enciclopedico L'Italin vivente: "Montoro Letteria, siciliana. Prose e poesie varie. Ė giovane e con lo studio riuscirà buona scrittrice" (Carpi 1878: 584)

3 Citiamo un brevissimo accenno, presente ne Il romanzo di Gino Raya, dove di Montoro si dice che "spaccò in due il secolo con Maria Landini" (Raya 1950: 217); il romanzo in questione sarà edito in effetti nel 1850. Ancora troviamo qualche riferimento a Montoro nella monografia di Marinella Fiume su Mariannina Coffa Caruso, ma solo in relazione a quest'ultima poetessa, per la quale Letteria aveva scritto una poesia in mortem (Fiume 2000: 357). Elena Sodini dà notizia della partecipazione della scrittrice messinese alla "Strenna femminile a profitto dell'Associazione Filantropica delle donne Italiane", edita nel 1861 (Sodini 2004: 348); Jole Calapso ci informa del fatto che Montoro scriveva pe la rivista "La donna", diretta da Gualberta Alaide Beccari, insieme ad altre scrittrici, per le quali "il desiderio d'affermazione personale e l'impegno civile qualche volta s'intrecciavano" (Calapso 1980: 45). Anche Gigliola de Donato, in Donna e società nella cultura moderata del primo Ottocento fa il nome di Letteria Montoro fra le collaboratrici di "La donna" (De Donato 1983: 92); Verdirame ( 2009: 59 n. 125) fa invece il nome della rivista genovese "La donna e la famiglia". di Cecilia Stazzone, ed accennato ad un'opera di Rosina Muzio Salvo, prosegue: "Lasciamo egualmente la signora Letteria Montoro, avvengacchè entrambe non siano nuove al pubblico dei lettori italiani, ma piuttosto presentiamo a questi Concetta Stazzone, e il suo romanzo Zelmira (Cattaneo 1925: 375). Ferdinando Bosio include una lirica della nostra sfuggente scrittrice in un'antologia, Le poesie di illustri italiani, corredando il testo con una breve frase: "e da Messina Letteria Montoro, di cui meglio è dir niente che poco" (Bosio 1865:19).

Avremmo preferito avere questo "poco", ma anche i contemporanei, come i critici odierni, si limitano a rapidi giudizi, che testimoniano comunque l'indubbia notorietà della scrittrice. ${ }^{4}$ In un articolo de "Il Poligrafo", a firma F. M. P., Montoro chiude l'elenco delle scrittrici siciliane, e si prevede per lei maggior fama se curerà la diffusione delle sue opere,

Ed anco noi abbiamo una giovane, la quale siederà non ultima fra queste valenti, se vinta la ritrosia, che le è propria, pubblicherà i suoi versi, dai quali l'Italia apprenderà di avere una novella poetessa in Letteria Montoro, che di presente, a quanto mi vien detto, lavora ad un romanzo contemporaneo (F.M.P. 1856: 316).

Non si tratta del già citato Maria Landini, edito nel 1850, ma di un altro lavoro, a cui ancora fa riferimento, anni dopo, Francesco Guardione: "Auguriamo che la gentildonna pubblichi il lungo romanzo, ch'è ricordo del nostro passato" (Guardione 1885: 333). Possiamo ipotizzare che l'espressione piuttosto generica alluda ad un'opera storica, o forse di ambientazione contemporanea ma riferita a recenti eventi sociopolitici siciliani, oppure italiani, secondo l'estensione geografica da attribuire all'aggettivo 'nostro'. Si riferisce probabilmente a questo stesso romanzo l'editore Treves quando, alla fine del 1861, pubblica un consuntivo della sua rivista, il "Museo di Famiglia", annunciando l'uscita di un nuovo romanzo di Letteria Montoro e un racconto di Rosina Muzio Salvo, "egregie scrittrici siciliane" (Grillandi 1977: 206). Si consideri che Treves aveva già pubblicato alcuni testi poetici di Montoro nel primo volume di "Museo di Famiglia" (1861), e inserirà l'anno dopo nel secondo un racconto della scrittrice, dal titolo "Lodovica Gerla".

4 Degno di nota il giudizio di Giuseppe Pitrè, che considera la produzione di Montoro, insieme a quella di altre scrittrici siciliane, come rappresentativa di un sottogenere di letteratura femminile circoscritto a livello regionale: "Dopo la perdita, ahi quanto dolorosa, della Giuseppina TurrisiColonna, dopo quella della cara fanciulla Lauretta Li Greci, questa della Muzio Salvo nonè lieve iattura per la Sicilia, dove poche fra le donne coltivano con onore le lettere. La Concettina Ramondetta-Fileti di Palermo, la messinese Letteria Montoro e la netina Mariannina Coffa-Caruso rappresentano ora la sicula letteratura femminile" (Pitrè 1868: 128) ". Francesco Guardione, storico e critico mazziniano, pubblica due poesie di Montoro nella sua Antologia poetica siciliana del secolo XIX, attribuendo, sia pure in modo non esplicito, una significativa valenza politico/ sociale alla produzione dell'autrice messinese: "Letteria Montoro ha cari versi, che riuniti formerebbero un elegante volumetto. Inoltre le leggiadre prose, dopo un romanzo dettato in giovinetta età, la fecero molto nota in Sicilia e nel Continente, e in tempi pericolosi la sua mesta espressione aveva un alto significato." (Guardione 1885: 333) 
Non è quindi ignota ai contemporanei quest'autrice pubblicata anche da Treves, su cui Cattaneo sorvola perché non c'è neanche bisogno di presentarla, che ha al suo attivo varie poesie - e ci si augura di vederle unite in una silloge -, di almeno due romanzi e alcuni racconti. ${ }^{5}$ Montoro, insieme ad altre valenti scrittrici siciliane (Rosina Muzio Salvo, Concettina Ramondetta Fileti, Mariannina Coffa Caruso), le quali “a ben far pongono l'ingegno, onora l'isola nativa, che bene a ragione si allieta de' generosi figli". Sono parole di Carmelo Pardi che, nei suoi Scritti vari, sottolinea l'importanza di questo "onorevole drappello delle nostre valorose" (Pardi 1871: 377).

E bisognava avere realmente coraggio per intraprendere la via della scrittura femminile nella Messina di primo Ottocento, dove si scontava una doppia marginalità rispetto al milieu culturale italiano: l'essere donna e l'essere siciliana. Quella in cui agisce e pubblica Montoro è un'area culturale problematica, distante dai centri nevralgici del dibattito intellettuale europeo, e tale da non consentire alle scrittrici di evidenziare una loro specifica fisionomia intellettuale, per la formazione letteraria superficiale e aspecifica riservata alle 'giovinette', le minime opportunità editoriali, la cogenza dei doveri familiari; infine il discredito in cui è posto qualunque tentativo, da parte di una donna siciliana, di acquisire visibilità sociale. Ne deriva una produzione sommersa, che spesso rimane inedita e diffusa solo in un circuito amicale. ${ }^{6}$ Quando una donna riesce

5 I testi poetici di Montoro non sono mai stati riuniti in volume e a questa dispersione alcun critici attribuiscono la scarsa notorietà dell'autrice. Forniamo un elenco, che non pretende di essere esaustivo, dei componimenti: Ad un giacinto, 1853 quartine, ne "La Missione delle donne", periodico diretto da Olimpia Saccati; Il pensiero dell'anima, 1853, pubblicato in una raccolta di Ferdinando Bosio del 1865, Le poesie di illustri italiani, poi nella Antologia poetica siciliana del secolo XIX (1885) di Francesco Guardione; La solitudine. Ottave a rima data, in "Eco Peloritano", Anno V, Serie II, fasc. 7, gennaio 1859, Un sospiro, in "Strenna femminile dell Associazione filantropica delle Donne Italiane", Torino, $1861_{i}$ Pel centenario di Dante Alighieri, 1864, in Alcune poesie di Mariannina Coffa Caruso da Noto e di Letteria Montoro da Messina, Palermo, Virzì, 1864 e nella Antologia poetica siciliana del secolo XIX (1885) di Francesco Guardione; Sospiro di una vergine candiota, in Candia: scritti in prosa e in verso, Messina, D'Amico, 1868; A Sofia giovinetta. Canzone, 1865, in Ghirlanda della Beneficenza, Messina, Stamp. Capra, 1872; Sulla tomba della Chiarissima Mariannina Coffa poetessa notina. Versi sciolti letti nell'Accademi radunanza straordinaria del 6 aprile 1878 nel Gabinetto Letterario Ibla Erea di Ragusa, Palermo, Virzì, 1878, Sul sepolcro del Sacerdote Francesco Montoro sua sorella Letteria, Messina, Tipografia del Progresso, 1886. Gaetano Oliva fornisce un elenco di poesie pubblicate in "La Favilla" di Palermo: Memorie, A Vincenzo Baffi, A Bellini, Amore, All'amico Carmelo Pardi, La preghiera dell'anima, L'usignolo, Al fiore del mio pensiero, Nella selva, Una preghiera di fanciulli nell'Asilo, Alla luna, Dimenticarti?, Nel mio balcone, Una memori dei tempi della speranza, Al mio fiore, Fantasia, Per un estinto fanciullo, Lucia, Per le nozze d'oro del Santo Padre Leone XIII. Anche i romanzi e i racconti appaiono su vari periodici; si salva dalla inevitabile dispersione il primo, pubblicato per una casa editrice palermitana: Maria Landini. Romanzo. Palermo, Clamis e Roberti, 1850; Lodovica Gerla, in "Museo di Famiglia", 1862; Silvia, racconto, Pubblicazione postuma in XXXIX Capitoli, nell'appendice del Giornale quotidiano "L'Ordine", Messina, 1898-99, Frammento di un romanzo inedito, in "Strenna veneziana", 1866.

6 E' del 1984 un saggio di Francesco Brancato sulla condizione della donna siciliana fra' $800 \mathrm{e}^{\prime} 900$ se il lavoro andrebbe rivisto alla luce delle recenti acquisizioni sulla 'presenza' femminile isolana in ambito letterario e sociale, soprattutto riguardo al periodo risorgimentale - ambito di ricerca a cui appartiene anche il presente lavoro - il saggio mantiene una sua validità nel definire le ragioni di una emarginazione pianificata, da parte delle classi dominanti maschili, della donna siciliana (Brancato 1984: 215- 252), per quanto sia documentata l'esistenza di un protagonismo femminile in ambiti di competenza tradizionali, anzi arcaici, quali il ristrettissimo spazio della famiglia (Resta 1983: 5-9).

$$
\text { Revista Internacional de Literaturas y Culturas, abril } 2017
$$

a pubblicare i suoi scritti il fatto viene percepito come eccezionale: Montoro, e altre intellettuali siciliane, sono definite con metafore belliche - il "drappello di valorose" immaginato da Carmelo Pardi -, e la loro letteratura viene identificata come altra rispetto a quella ufficiale; Pitrè e Di Pietro, come abbiamo visto, definiscono un vero e proprio canone di letteratura femminile siciliana. Si tratta quindi di donne 'ribelli', che oppongono alle regole dell'universo maschile una propria visione del mondo, anche quando essa non si affranchi interamente da modelli maschili prestigiosi (Calapso 1980).

La produzione di queste scrittrici è stata fatta oggetto di indagine critica soprattutto negli ultimi venti anni, quando la letteratura femminile siciliana dell'Ottocento arriva ad essere considerata un campo fertile di analisi, anche per definire con maggiore esattezza l'apporto della Sicilia al discorso culturale italiano ed europeo. ${ }^{7}$ La maggior parte delle colleghe di Montoro appartiene geograficamente all'area palermitana, o comunque gravita intorno a Palermo; ma anche Messina, la città più 'continentale' della Sicilia, strettamente collegata alla capitale del regno, ad una Napoli verso cui letterati messinesi tendevano per pubblicare su periodici a rilevanza nazionale, offre interessanti opportunità agli intellettuali, a qualunque sesso appartengano. In questo quadro bisogna leggere l'attività letteraria di Letteria, che non è così esigua come alcuni critici hanno sottolineato, ma soprattutto si presenta diversificata e tale da superare il ristretto ambito cittadino: Montoro pubblica spesso a Palermo, per editori ed in riviste; oltre lo Stretto ottiene l'inserimento nella "Strenna filantropica", progetto fortemente voluto dalla duchessa Felicita Bevilacqua, che aveva richiesto per la sua attuazione un fitto scambio epistolare fra intellettuali donne di ogni parte d'Italia (Sodini 2004: 348). A Messina inoltre opere della scrittrice sono inserite in volumi antologici di portata nazionale: la "Ghirlanda della beneficenza", come in precedenza la "Strenna filantropica", riunisce testi poetici di autrici non solo siciliane ma italiane; "Candia: scritti in prosa e in verso" è una pubblicazione di grande rilevanza politica, finanziata dal comitato filellenico messinese per aiutare i combattenti cretesi del 1866.

La produzione poetica di Letteria oggi appare datata, perché improntata a quel leopardismo 'minore' così frequente in area meridionale; in esso l'enunciazione del dolore come fatto costitutivo dell'essere umano appare irrelato alla riflessione sulla 'natura matrigna', e sul senso ultimo della sofferenza, che caratterizza il discorso di Leopardi e trova invece una risposta consolante nella fede religiosa. Di un certo

È quasi superfluo sottolineare che l'interesse per le letterate siciliane è derivato anche dall'evoluzione dei gender studies verso modalità di approccio più complesse e produttive, grazie alla valorizzazione del contesto storico e sociale come elemento fondamentale da cui nasce il discorso letterario delle donne scrittrici; ci riferiamo, ad esempio, all'autobiographical criticism, che recupera nell'analisi della produzione di una autrice, la sua esperienza personale e il suo vissuto sociale "Il discorso diviene riconducibile alla concretezza delle donne, al piano del vissuto, a un contesto socioculturale e non più all'astrazione dell'essenza" (Gaieri 2002: 251). 
interesse solo Il pensiero dell'anima (Montoro 1885), che pone il problema della trascendenza come illusione necessaria all'uomo: il lungo componimento, una canzone libera in endecasillabi e settenari, presenta riconoscibili influssi leopardiani non solo a livello metrico ma lessicale; tenta inoltre un discorso lirico/ filosofico basato su di un'impalcatura logica ben visibile nella predominanza dei nessi casuali e temporali. ${ }^{8}$

Bisogna dire che, se le testimonianze coeve apprezzano in Montoro la poetessa considerano però con maggiore interesse il già citato Maria Landini, l'unico suo romanzo edito integralmente. Il fatto che sia una donna a scrivere e pubblicare romanzi è, per la realtà siciliana, molto significativo; in una situazione culturale arretrata come quella messinese, quando ancora la maggior parte degli intellettuali si affidano al testo poetico come mezzo di espressione principale, servendosi fra l'altro di strutture classiche ed un lessico arcaico, possiamo annoverare solo tre romanzi: un soggetto filellenico non originale, La tomba di Marco Botzari di Francesco Soraci, traduzione di un romanzo di Camille Paganel; Gli Albigesi di Giuseppe La Farina, denso romanzo storico con un occhio alla contemporaneità, poiché mette in discussione il ruolo della Chiesa nel Risorgimento, e appunto Maria Landini. Sorprende che arrivi proprio dal margine dell'intellettualità femminile la proposta del genere romanzo, caratterizzato dalla plurivocità, da un approccio al reale meno mediato rispetto al discorso poetico, dalla possibilità di utilizzare uno stile concreto e più vicino, per sintassi e termini, alla lingua viva, infine da una nuova concezione della storia. Un genere che appartiene alla modernità, ma non alla Sicilia classicista; la 'ritrosa' Letteria opta quindi per modalità

8 Il componimento traccia il percorso conoscitivo dell'io lirico, da un primo momento positivo, di ingenua e festosa accettazione dell'immortalità e dell'esistenza dell'anima - troviamo precis reminiscenze da A Silvia ("Te scopo de la vita,/ quando d essa mavvidi, idoleggiai./ Per te la terra, ciel, l'astro del giorno, / l' lintera natura benedissi./Come il cor ti sentia! Quali diletti/L'immagin tua m porse!/ Che speranze, che affetti..."), alla disillusione ("ahi presto il mondo/ Al cor mancava"); infine labbandono totale dei "dolci inganni", 1 "crudi lampi del vero", la conseguente incomunicabilità con gli altri e con il mondo ("desolato ovunque il guardo io giri/ su quest'orrida landa, / ove alla mente e al cor nulla risponde"). Ma, nonostante tutto, la poetessa non rinuncia alla convinzione dell'esistenz dell'anima: "tu supremo desio, /primo sospiro d'ogni cor gentile, / nell'estasi del pianto / sol tu più cara del morir mi sei". (Montoro 1885: 335-336). Insomma ci sono tutti i materiali da costruzione dell'ideologia leopardiana, e sono presenti anche componenti stilistiche riconoscibilissime, qual l'insistito procedimento anaforico dei nessi disgiuntivi e correlativi; le esclamative patetiche introdotte dall'aggettivo o pronome "Quale / quali"; molti elementi di lessico e un affine andamento sintattico; manca però la volontà di portare il discorso alle ultime conseguenze. Montoro condivide nelle linee generali la poetica doloristica leopardiana poiché la sente affine alla propria esperienza biografica, ma le rimane estranea la complessità del pensiero di Leopardi e soprattutto il suo laicismo.

La predilezione di Montoro per Leopardi si deve inquadrare nel più generale interesse che la Sicilia riserva al poeta recanatese: nel 1835 alcuni noti intellettuali messinesi ( Felice Bisazza, Giacomo Rol) si recano a Napoli proprio per conoscere di persona lo scrittore "avendo letto ed ammirato Messina le splendide liriche leopardiane" (Perroni Grande 1904: 177). La tipografia palermitana di Francesco Spampinato aveva infatti pubblicato nel 1834 i "Canti" leopardiani, dall'edizione Piatti del 1831, e il volume era stato recensito da Pompeo Insenga nel "Giornale di scienze lettere e arti per la Sicilia", XIII, 1835, vol. 49, pp. 97-100; il poeta e letterato siracusano Tommaso Gargallo invita Leopardi all'Università di Palermo per un corso di lezioni, possibilità forse sfumata per opposizione del governo borbonico. di espressione meno usuali, e la professione di modestia contenuta nella prefazione sembra voler attenuare, ma non cancella, la portata innovativa della scelta. ${ }^{9}$

Maria Landini racconta l'intricata vicenda di una ragazza, Maria, che abbandona la propria casa e i parenti per evitare l'unione con un personaggio ricco e malvagio, il barone Summacola; nella sua fuga rocambolesca viene aiutata dalla famiglia di Roberto Altieri, danneggiata dallo stesso barone..$^{10}$ Nella trama sono facilmente riconoscibili elementi manzoniani, per quanto ribaltati di segno: il matrimonio non è il fine ultimo della vicenda ma un male da evitare; la protagonista non accetta le situazioni predisposte da altri, ma oppone ad essi la propria libera volontà. Maria sembra essere un'anti-Lucia, anche se il suo rifiuto di lasciarsi guidare dall'ipocrita logica borghese degli adulti è condotto in nome di un'onestà e purezza interiore che, in altre forme, troviamo anche nell'eroina manzoniana. ${ }^{11} \mathrm{E}^{\prime}$ essenziale il fatto che la protagonista si mantenga distante da un atteggiamento acquiescente, tradizionalmente femminile;

9 Afferma infatti Letteria nella prefazione intitolata "Due parole al lettore", che nelle pagine che seguono "non troverai sfoggio di erudizione o di stile, perché non ho consultato alcun libro, né mezzi ebbi allo studio" (Montoro 1850: 7)

10 La trama completa è la seguente: Roberto Altieri soccorre con la figlia Giulia una giovane sconosciuta, che si saprà poi essere Maria. La coppia è affitta perché lálto fighio, Edoardo, è stato arrestato da un malvagio magistrato, il barone Summacola; la madre Adelia ne muore di dolore. Inutilmente gli Altieri avevano cercato di intercedere presso la corrotta nobilà del luogo; neanch il pontefice aveva dato loro ascolto. La storia di Maria e le vicende degli Altieri sono connesse in vario modo: Edoardo aveva conosciuto casualmente Maria e se ne era innamorato; la ragazza a sua volta era stata promessa dagli zii proprio a Summacola, ma temendo il barone, del quale avvertiva la doppiezza, era fuggita nella notte. Maria era gia sradicata dal proprio contesto familiare di origine perché figlia di un esule, messo al bando per aver aiutato un uomo ingiustamente accusato; alla morte del padre, impazzito dal dolore, Maria era andata a vivere a Roma dalla zia. Nella sua fuga che raccontera agli Altieri, la ragazza incontra un vecchio saggio; questi le fornisce una scorta cu affidarsi durante il viaggio, un uomo sfregiato di nome Lorenzo, che e in realta Enrico, amico di Edoardo, riuscito a sfuggire all'arresto: la cicatrice deriva da uno scontro con dei banditi. Maria e Lorenzo s'imbattono in un gruppo di armati, ma la ragazza riesce a fuggire, arrivando stremat in un cimitero dove la trovano gli Altieri. Mentre Edoardo soffre nella terribile prigione di Caste Sant'Angelo Enrico, sotto le spoglie di Lorenzo, elabora un piano per farlo fuggire, convincendo un carceriere. Dietro tutte le mosse dei giovani ci sono i consigli del vecchio saggio, che altri non è che il nonno di Enrico, Giovanni Martelli, un uomo giusto costretto all'esilio. Edoardo ed Enrico, ormai liberi, si recano in Francia; a Roma Roberto si batte in duello con Summacola e lo sconfigge; questi si pente e rivela di aver perseguitato gli Altieri poiché aveva amato senza speranza la moglie di Roberto, Adelia. Nella conclusione Roberto e Giovanni Martelli si ritrovano, gli esuli tornano, e il magistrato si ritira in eremitaggio.

11 I parenti si mostrano del tutto propensi ad un'unione della povera orfana con un personaggio benestante, anche se potenzialmente malvagio; tanto, afferma la zia ipocritamente, "coi tuoi consig e col tuo esempio lo trarresti al sentiero d'onore" (Montoro 1850: 83). La pressione psicologica della zia, i suoi insistenti ricatti sentimentali, così violenti che Maria arriva a temere non l'ira della donna ma piuttosto "le sue parole amorevoli" (Montoro 1850: 81), il richiamo subdolo alla duplice autorità del padre morto e di un Dio benedicente l'unione, immediatamente smascherato da Maria ( "Ah! It padre avrebbe interrogato sì i miei palpiti, ma non ha mai pensato dirigerli!"; Dio! Tu il cuore creas libero"), ricordano molto da vicino la monacazione forzata di Gertrude, ma anche in questo caso il romanzo di Montoro riprende alcuni elementi della vicenda manzoniana ma al tempo stesso ad essa si oppone specularmente: Maria ha infatti la forza di reagire. Se leggiamo Maria Landini anche come riscrittura de I Promessi Sposi, è significativo il rapporto critico e a volte conflittuale con il modello 
l'unione d'interesse è vista come "sacrificio di una vittima", "ferale olocausto"; una violenza terribile che, per quanto ammessa dalla società, turba l'equilibrio naturale ed è infatti accompagnata, nel giorno delle nozze, dallo scatenarsi degli elementi: "nel sibilo del vento, che sordo e sinistro perturbava l'aere, io sentiva il preludio di eterna maledizione alla mia viltà. Io vile! Io l'obbrobrio di me stessa!... non sarà mai! " (Montoro 1850: 86). Nell'ottica ribellistica di Maria una simile sopraffazione richiede una ferma opposizione e un grande coraggio: la successiva fuga, dalla connotazione insieme gotica e favolistica - le tenebre, lo smarrimento nel bosco, il senso di rovina e distruzione che emana dal paesaggio naturale -, simboleggia l'ingresso in un altro mondo, una tappa cruciale di morte/ rinascita della protagonista la quale, abbandonato il cerchio sicuro ma angusto della casa, può fare ora esperienza del mondo. ${ }^{12}$ Anche in questo il personaggio appare diverso rispetto ad una Lucia legata al cronotopo della casa, e piuttosto vicino al percorso accidentato ma formativo che compie Renzo; da questo momento infatti Maria inizia un viaggio attraverso la campagna italiana tormentata da miseria e violenza, dove l'illegalità è imperante: un mondo abitato da mendicanti e briganti, nel quale si vive in una condizione di costante pericolo. ${ }^{13} \mathrm{La}$ realtà rurale è presentata come un altrove, completamente distante dal passato di Maria, ma non per questo un mondo estraneo: la ragazza infatti conclude la fuga in ambienti accoglienti - la capanna di Martelli - dove gli arnesi di lavoro sono indizio di onestà

12 "Agitata da violente vertigini sentiva venir meno i miei sensi, e non discernea che la confusione di un sogno [...]; un fremito mortale mi scuote le fibre [...]. M'avvio tacita e furtiva per le scale, $\mathrm{m}$ da lì odo calpestio ... misera me! Mi arresto inosservata, penso ... havvi nella casa un'uscita segreta vi traggo per buio ed angusto corridoio: la porta e attraversata da una spranga ... Dio, soccorso! Che fare? ... coraggio! Mi pruovo piano di trarre quella spranga dai buchi, le mani tremano, essa stride, sono perduta! ... ritta mi sto li, trattenendo financo il respiro, ma era silenzio, nessuno avea avvertito quel rumore: tento di nuovo, ecco sbarrata la porta ... che oscurità intensa! Qual tempesta di affetti in me! ... brancolando discendo gli ineguali scalini, balzo di terrore ad ogni passo, disserro a stenti il portone, ed eccomi nella strada [...]. Il vento imperversa orribile, e l'aere suona pianto! Le tenebr s'addensano sul mio capo" (Montoro 1850: 87, 89). “Da circa due ore io camminava per quella strada e grosse gocce d'acqua sentia spesso sul volto. Una stella cercavano invano gli occhi miei; un negro manto vestia la volta dei cieli, e gravando sulla faccia della terra mi copria di suo orrore come la notte del sepolcro [...]. La via ch'io percorrea non la vidi più dritta e piana ma scoscesa, m'incespava nei suoi sterpi, pure il breve ma oramai speso lume dei lampi guidava i miei passi per istretto e irregolare sentiero (Montoro 1850: 91). Si noti che il comportamento di Maria, per quanto metta in discussione le norme sociali, è additato come exemplum dai personaggi positivi della vicenda, Roberto Altieri esclama infatti, dopo aver ascoltato il racconto della fuga: "Oh, anche per mal'intesa affezione si domanda la schiavitù dell'affetto! ... M'interessa l'udirvi, o giovinetta; Se per poco tutti vi somigliassero!" (Montoro 1850: 89).

13 Anche Lucia fugge, ma è condotta da altri, mentre Maria affronta da sola i pericoli della notte, decidendo oltretutto di abbandonare i centri abitati per maggior sicurezza. Ė proprio la centralità della fuga, motore narrativo del romanzo di Montoro, a qualificarne la portata innovativa, la volontà di mettere in discussione il canone letterario di primo Ottocento, secondo il quale "la casa non è solo luogo di centralità narrativa, ma anche ideologica, perché è espressione di norme maschili, e da ciò deriva l'inquietante per le donne, con rimozioni, crepe, fughe. Nell'Ottocento la casa appare dominante tra le forme di organizzazione spaziale e sociale dei modelli culturali patriarcali: la donna è relegata nella casa, che offre un alone di sacralità purché lei ne rispetti il confine" (Barbarulli-Brandi 2004: 417). povertà, ed il rischio non proviene certo dai banditi, anch'essi rifiutati da una società che li ha costretti ai margini; le parole del vecchio saggio, l'esule Martelli appunto, scardinano, sia pure in modi ingenuamente populistici, il sistema di valori dominante: Sì, figlia mia, l'infelice ama l'infelice: quelli non avrebbero disfogato in voi l'odio che 1 martira, si lo stesso delitto pur li raccapriccia .... anche il malvagio sente in sé quel germe di (a) ed essi amano il vizio per sola ostentazione (Montoro 1850: 95).

L'allusione ad un sistema feudale improduttivo e vessatorio non è qui particolarmente evidente, ma il discorso va inquadrato nel contesto del romanzo; nelle prime pagine infatti, quando si racconta la storia di Roberto e del suo matrimonio con una nobile persiana, Adelia, il padre della sposa aveva descritto la situazione negativa del suo paese, la Persia:

Oppressa dagli orrori d'una barbara feudalità, senza forza e concentrazione $\mathrm{d}$ potere, senza istruzione né progresso, ella è abbandonata come schiava ai capricc degli uomini [...]. Ma il mio peggior dolore è nel considerare questo popolo, su cui passano i secoli, e non un pensiero, non un palpito ne contrassegna il passaggio: vero frammento della massa orientale, l'inerzia sembra essere l'elemento e lo scopo del suo vivere, il riposo l'unica sua felicità, fosse pur quello della morte! [...] Sepolta nelle rovine di sua gloria, giace sulla terra, grave, stazionaria, ed immensa (Montoro 1850: 25).

In epigrafe una citazione dalla canzone All'Italia di Leopardi orienta l'interpretazione del testo in senso politico, ${ }^{14}$ l'autrice utilizza l'immagine di una nazione lontana ed esotica per permettersi una dura critica alla sua terra: statica, arretrata, inerte.

D'altra parte la causa originaria del ribellismo di Maria è in sé politica: la ragazza infatti è figlia di un'esule, già da tempo quindi posta ai margini della società. La fuga è allora un'occasione per osservare la realtà da vicino, con l'occhio critico dell'outsider, che scorge in tutta la sua drammaticità la continua violenza sui deboli e la sopraffazione dei potenti senza regola né legge. ${ }^{15}$ Un'esperienza dolorosa ed al tempo stesso conoscitiva: durante la sua iterata e angosciosa fuga Maria sviluppa una coscienza

14 "Chi di te parla o scrive, / che rimembrando il tuo passato vanto/ non dica: già fur grande, or non è quella? Leopardi" (Montoro 1850: 23)

15 Durante la fuga Maria incontra una donna ridotta a mendicare perché il marito contadino era stato ucciso dall'eccessivo lavoro: "Ella piangea stringendosi al seno quel pargoletto; la sua figura era squallida e trista, le sue vesti cenciose, e quel bambino parea succhiare invece del latte il dolore della madre sua! Ella mi vide, e mi si avvicinò mostrandomi il volto pallido e magro del figlio, e stendendo la scarna mano con tal atto da ferirne il cuore! ... Scoppiando in pianto la tapina mi raccontò la cagione di sua miseria; mi raccontò com'era stata sposa ad un contadino, il quale col suo stentato sudore procacciava appena un nero pane onde disfamare la meschina famiglia, e come finalmente morto per esorbitanti travagli il marito, ell'era costretta ad accattare un pane elemosinando!!" (Montoro 1850: 102).

Rista Internacional de Literaturas y Culturas, abril 2017 
del proprio destino come diverso, forse non immediatamente coincidente con quello di moglie e madre. Troviamo inoltre, nelle parole della giovane, una connessione fra l'intensa sofferenza che vive su di sé o individua nel reale e l'ideologia democratica e risorgimentale: prova dolore chi è escluso dalla società per le sue aspirazioni libertarie, o chi è oppresso dalla prepotenza dei 'signori', a qualunque classe appartenga. ${ }^{16}$

La vicenda in effetti si mette in moto proprio per la doppia violenza del barone Summacola, nei confronti della famiglia Altieri e di Maria stessa; una violenza derivata da una feroce volontà di sopraffazione. Nelle azioni della protagonista confluiscono quindi le due linee ideologiche del romanzo: la condizione di esule, innestata sulla marginalità costitutiva del ruolo femminile, si potenzia grazie ad una percezione dolorosa del mondo di matrice vagamente leopardiana, ed all'esperienza formativa del viaggio/fuga in luoghi ignoti, determinando in Maria una capacità inedita di fronteggiare la realtà, e di valutarla facendo emergere gli aspetti problematici della società contemporanea. ${ }^{17}$ Importante in questo quadro l'immagine noir dei personaggi appartenenti alla nobiltà o al clero, quindi alla classe dominante: ambigui e corrotti, questi uomini potenti indossano una maschera di perbenismo, ma di fatto utilizzano la loro posizione di dominio per soddisfare sordidi interessi personali. ${ }^{18}$ Alleati fra loro,

16 Il leopardismo attenuato di Montoro, come focalizzazione del dolore nell'esperienza umana, trova quindi all'interno del romanzo una sua specifica funzione e spessore sociale e politico, rientrando in un quadro ideologico più generale che è stato evidenziato da recenti studi: I'insistenza sul martirio e sulla pena è infatti elemento costitutivo dell'immaginario patriottico, e si pone fra l'altro l'obiettivo di recuperare il dolore delle fasce più deboli della società - esuli, donne, inserendole in un quadro di obiettivi comuni a cui aspirare (Banti, 2000; Banti, 2011). Vale la pena osservare che l'attenzione per la dimensione politico/risorgimentale sottesa alle vicende raccontate non è usuale per il periodo considerato, e per questo genere letterario; il feuilleton italiano tende infatti ad accentuare gli aspetti (a) Reim 2000: 21).

17 Possiamo utilizzare, per spiegare il senso dell'esperienza compiuta da Maria, le parole di Silvana Serafin sulla valenza conoscitiva del viaggio di migrazione in una scrittrice del Novecento, Syria Poletti: "In questo andare l'individuo compie una spoliazione di sé, esplorando gli abissi della coscienza, entrando nel nucleo del sapere, dove la rivelazione degli aspetti più oscuri, permette di eliminare sovrastrutture limitative alla crescita interiore. Nell'emigrazione, infatti, paradigma di avventura e di mobilità, si condensa un momento essenziale di transizione e di evoluzione del percorso esistenziale." (Serafin 2008: 151)

18 Summacola in pubblico mostra un aspetto saggio e dignitoso: "l'alta sua fronte splende di nobili pensieri, e le sue labbra sono atteggiate ad un benevolo sorriso"; in privato si trasforma nel tipico vilain dei romanzi popolari: "allora getta lungi da sé quel velo che ipocritamente l'avviluppava, prorompe in bestemmie, i suoi occhi lanciano velenosi sguardi come ambissero la potenza di inabissare l'umanità la sua fronte dapprima serena ecco adesso corrugarsi, e ripullulare in essa avide le idee del delitto, del tradimento, ed il sorriso cambiarsi in un sogghigno colmo di fiele e di livore mortifero" ( Montoro 1850: 29-30). Anche nei personaggi manzoniani negativi di classe agiata - Don Rodrigo, Gertrude troviamo una simile caratterizzazione, ma in Maria Landini il noir è cifra compositiva del discorso, ed interessa anche il paesaggio che la protagonista si trova a percorrere, in una definizione del Male come elemento pervasivo dell'intera realtà; con esso la ragazza coraggiosamente si confronta, trasformandolo in strumento ermeneutico per comprendere il mondo che la circonda ma anche se stessa, i propri obiettivi e limiti. Le stesse modalità rappresentative troviamo nel romanzo gotico al femminile di Ann Radcliffe, dove il paesaggio, connotato dal sublime orrifico, "ha in sé i segni della

$$
\begin{aligned}
& \text { Revista Internacional de Literaturas y Culturas, abril } 2017 \\
& \text { ISSN: } 1885-3625
\end{aligned}
$$

il barone Summacola, il Cardinale Ministro di Polizia, lo stesso pontefice, appaiono al lettore circondati dal lusso ma al tempo stesso avvolti dalla penombra, in scenari dal cromatismo cupo e livido; simboli evidenti della loro personalità impura. ${ }^{19} \mathrm{Se}$ la divisione manichea in buoni e cattivi, ed alcuni elementi della struttura - intreccio di vicende, con meccanismi di sospensione per tener desta l'attenzione, ripetitività di eventi e situazioni, linguaggio sintatticamente semplice con impasto lessicale di residui aulici e popolarismi - sembrano assegnare Maria Landini al genere de romanzo popolare, l'opera invece non è facilmente classificabile: non propone infatti facili soluzioni al male nel mondo, quali l'eroe giustiziere superomistico che risolve gli intrighi del feuilleton; ${ }^{20}$ presenta al lettore una protagonista femminile che si rifiuta di rientrare nei canoni della 'fanciulla perseguitata' di tanti testi romantici, ma porta avanti, sia pure fra dubbi ed esitazioni, una sua battaglia personale, ${ }^{21}$ e l'autrice sfrutta la plurivocità inerente al genere per descrivere il momento cruciale di ribellione $\mathrm{d}$ Maria attraverso i suoi occhi e le sue parole, mediante un processo di focalizzazione interna. Assolutamente non consolatorio ma piuttosto problematico, questo romanzo storico-contemporaneo intessuto di elementi popolari ha il merito di fornire uno

natura più intima degli uomini, le loro pulsioni segrete, i loro sogni [...]; il paesaggio, nei suoi più mutevoli aspetti, accompagna la protagonista nel suo viaggio alla ricerca di sé, della sua più intima natura" ( Rosa Scrittori 2004: 353).

19 "Il pallido chiarore della lampada si rifletteva incerta sugli apparti violacei della stanza, ove assiso sur una poltrona stava il Cardinale Ministro di Polizia vestito della porpora"; "Coverto dell'ermellino stava il Pontefice assiso sur una specie di trono, coi piedi poggiati sopra un cuscino di di fignificativa la figura del pontefice, che di fronte alle donne imploranti non trova di meglio che rispedirle al Ministro di Polizia sulla causa di queste infelici presso il ministro. Così il Pontefice comode questuanti. "Accudite va delle donne quel rivolo di speranza che si intromette a prolungare il dolore" (Montoro 1850: 45). La cattolica Montoro mette in scena un papa complice di funzionari corrotti, evidenziando una volonta di denuncia sociale ed una 'cattiveria rappresentativa' - nota formula di Romano Luperini, riferit in più occasioni non solo a Verga (vedi il recente Luperini 2005: 83, 164), ma anche a Manzon -, che forza i limiti del "vero" romantico.

20 Ci riferiamo ovviamente al discorso di Eco 2001.

21 La "fanciulla perseguitata" per eccellenza nel periodo romantico è proprio la Lucia manzoniana, con la quale la Maria di Montoro intrattiene una relazione complessa di affinità/ differenza/ opposizione; è vero che la protagonista de I Promessi Sposi non può essere soltanto ricondotta a questo antichissimo stereotipo - che data al romanzo ellenistico e si diffonde grandemente nella letteratura e cultura popolare europea - poiché è personaggio di ben altra complessità (Raimondi 1974) ma un elemento accomuna Lucia e le altre "damsel in distress": una sostanziale passività, o un attivismo comunque eterodiretto, in genere di matrice religiosa.Maria invece agisce in totale autonomia, senza alcuna guida - ha rifiutato, si è detto, i consigli e le proposte degli adulti - e senza il conforto di ideologie e schemi comportamentali condivisi dalla comunità in cui vive, secondo la quale l'obiettivo primario della donna è il matrimonio, ed il prestigio sociale dato dall'unione con un uomo influente. Si potrebbe definire Maria, con lieve forzatura, una "personaggia", utilizzando il neologismo coniato dalla Società Italiana delle Letterate, cioè una figura che nasce in un preciso tempo storico e ambiente sociale ma rigetta gli stereotipi e rinnova gli schemi del contesto culturale e della realtà esistenziale in cui si trova ad operare (Mazzanti et al 2016). In realtà la protagonista mostra una consapevolezza ancora embrionale della trasgressione insita nei propri comportamenti, e nella conclusione si sposa, regolarmente, con l'uomo che ama. Ma più che l'approdo conta il percorso, e la sfida alle convenzioni. 
spaccato della vita sociale del tempo, sia pure con i limiti dati da un codice linguistico legato alla retorica risorgimentale e talvolta poco efficace a livello espressivo. ${ }^{22}$ Dietro il personaggio di Maria, coraggioso ed in grado di sfidare le convenzioni, è agevole rintracciare la fisionomia dell'autrice, che condivide con la sua protagonista la dolorosa esperienza dell'esilio, ed una conoscenza più profonda delle cose e degli uomini; nel romanzo tale 'cognizione del dolore' e dell'emarginazione si converte in capacità di agire nel reale, e di vederlo senza illusioni; nella vita di Letteria diventa, semplicemente, scrittura.

La produzione letteraria di Montoro presenta quindi sicuramente elementi inusuali, sperimentali, innovativi: l'elaborazione di un romanzo popolare in cui avventura e realismo si coniugano offrendo al lettore uno spaccato della realtà meridionale alle soglie dell'Unità caratterizzato da una discreta efficacia rappresentativa; una figura di eroina inedita e propositiva; l'utilizzo di un immaginario gotico che sembra interpretare le istanze visionarie del Romanticismo nordico; infine la percezione di un disagio sociale autenticamente sofferto, e innestato senza cesure nell'immaginario risorgimentale, per cui bandito, esule, giovani donne vivono una stessa esperienza di esclusione, e ambiscono ad una eguale volontà di riscatto.

Da un estremo geografico, la Sicilia, e da una condizione sociale di per se stessa emarginata quale poteva essere quella della donna non sposata, che oltretutto ambiva ad essere scrittrice, che infine coltivava la sua vocazione in momenti residuali della propria attività di sorella, amica, confidente, ai margini del proprio quotidiano, emerge quindi un pensiero e un'opera di sicuro interesse, e tale da fornire spunti di riflessione per rivedere la storia vulgata di una Sicilia di primo Ottocento: isola al quadrato, separata dall'Europa, ma al tempo stesso grande periferia che può ambire, in qualche caso, ad acquisire una sua centralità.

\section{RIFERIMENTI BIBLIOGRAFICI}

Alfieri, G., "La lingua di consumo", en L. Serianni, P. Trifone (eds). Storia della lingua italiana. Scritto e parlato.,Vol. 2, Torino, Einaudi. 1994, pp.161-235.

Attard, G., Messinesi insigni del secolo XIX sepolti al Gran Camposanto. Epigrafi - Schizzi biografici, G. Molonia (ed.), Messina, Società Messinese di Storia Patria. 1991.

Banti, A. M., La nazione del Risorgimento. Parentela, santità e onore alle origini dell'Italia unita, Torino, Einaudi, 2000.

22 Sulle caratteristiche strutturali e linguistiche del romanzo storico/contemporaneo e popolare utile consultare rispettivamente Ganeri e Alfieri 1994: 161-235. La lingua di Montoro è quella di mol romanzi ottocenteschi, appesantita da detriti aulici che stridono con il registro medio dell'insieme da un'aggettivazione ridondante e anteposta al nome, dalla presenza di elementi morfologici desueti. quali ad esempio l'imperfetto di prima persona con uscita in -a. (Bricchi 2000)

Revista Internacional de Literaturas y Culturas, abril 2017 ISSN: 1885-3625
Banti, A. M., Sublime madre nostra: la nazione italiana dal Risorgimento al fascismo, Bari, Laterza, 2011.

Barbarulli, C., y Brandi, L., "Appartenenze, resistenze e transiti: nel riflesso inquietante dello spazio domestico", T. Agostini et al. (eds.), Lo spazio della scrittura. Letterature comparate al femminil., Atti del IV convegno della Società Italiana delle Letterate, Venezia, Fondazione Giorgio Cini, 2004, pp.417-8.

Bosio, F., Poesie, Milano, Guigoni, 1865.

Brancato, F., "La donna tra Otto e Novecento in Sicilia", Archivio Storico Siciliano. X: 1984. pp. 215-252.

Bricchi, M., La roca trombazza. Lessico arcaico e letterario nella prosa narrativa dell'Ottocento italiano, Alessandria, Edizioni dell'Orso, 2000

Calapso, J., Donne ribelli: un secolo di lotte femminili in Sicilia, Palermo, Flaccovio, 1980.

Cattaneo, C., "Sul romanzo delle donne contemporanee in Italia", Il Politecnico, XVIII: 89-112. Poi in Opere edite ed inedite. Scritti letterari, Agostino Bertani ed., Firenze, Le Monnier, 1925, pp. 358-389.

Carpi, L., L'Italia vivente: aristocrazia di nascita e del denaro-borghesia-clero burocrazia; studi sociali, Milano, F. Vallardi, 1878.

De Donato, G., "Donna e società nella cultura moderata del primo Ottocento" La parabola della donna nella letteratura italiana dell'Ottocento, Gigliola De Donato ed. Bari, Adriatica editrice, 1983. pp.11- 96.

Di Pietro, G., Illustrazione dei più conosciuti scrittori contemporanei siciliani dal 1830 a quasi tutto il 1876, Palermo, Amenta. 1878.

Eco, U., Il superuomo di massa. Retorica e ideologia del romanzo popolare, Milano, Bompiani, 2001 (1976).

Fiume, M., Sibilla arcana: Mariannina Coffa (1841-1878), Caltanissetta, Lussografica, 2000

F. M. P., “Corrispondenze dalla Sicilia”, Il Poligrafo. Vol. 2, 1856, pp.314-319.

Gaieri, E., "Femminismo e 'gender studies", Letteratura comparata. Armando Gnisci ed. Milano, Bruno Mondadori, 2002, pp. 235-264.

Ganeri, M., Il romanzo storico in Italia: il dibattito critico dalle origini al postmoderno, Lecce, Manni Editori, 1999

Greco, O., Bibliobiografia femminile italiana del XIX secolo, Venezia, presso i principali librai d'Italia, 1875.

Grillandi, M., Emilio Treves, Torino, U.T.E.T., 1977.

Guardione, F., Antologia poetica siciliana del secolo XIX, con proemio e note, Palermo, Tempo, 1885. 
Guardione, F., Poeti siciliani del secolo diciannovesimo, Palermo -Torino, Carlo Clausen, 1892.

La Corte Cailler, G., La donna nella beneficenza in Messina dal 12 al 19 secolo., Messina, D'Angelo. 1914

Luperini, R., Verga moderno, Bari, Laterza, 2005.

Montoro, L., Maria Landini. Romanzo, Palermo, Clamis e Roberti, 1850.

Montoro, L., "Il pensiero dell'anima". Francesco Guardione ed. Antologia poetica siciliana del secolo XIX, con proemio e note, Palermo, Tempo, 1885, pp. 333-336.

Montoro, L., Sul sepolcro del sacerdote Francesco Montoro, sua sorella Letteria., Messina, Tip. del Progresso, 1886

Oliva, G., Annali della Città di Messina (continuazione all'opera di Caio Domenico Gallo) con cenni biografici dei cittadini illustri della seconda metà del secolo 19, Vol. 8 , Messina, Società Messinese di Storia Patria, 1954. pp. 292-293.

Pardi, C., Scritti vari, Palermo, Tipografia del Giornale di Sicilia, 1871.

Perroni Grande L., “Giacomo Leopardi e Giacomo Rol”, Archivio Storico Messinese. Vol. 5, 1904, pp. 177-178.

Pitrè, G., Nuovi profili biografici di contemporanei italiani, Palermo, Tip. A. Di Cristina, 1868

Raimondi, E., Il romanzo senza idillio. Saggio sui Promessi Sposi, Torino, Einaudi, 1974.

Raya, G., Il romanzo. Milano, Vallardi, 1950.

Reim, R., I vili godimenti: storie di peccato e perdizione nel romanzo d'appendice italiano, Roma, Robin, 2000.

Resta, G., "Prolusione”, S. Zappulla Muscarà (ed.), Letteratura siciliana al femminile: donne scrittrici e donne personaggio. Atti del convegno nazionale di studio Misterbianco 1-3- dicembre, Caltanissetta- Roma, Salvatore Sciascia Editore, 1983, pp. 5-9.

Scrittori, A. R., "La geografia interiore del racconto gotico di Anne Radcliffe", T. Agostini et al. (eds.), Lo spazio della scrittura. Letterature comparate al femminile. Att del IV convegno della Società Italiana delle Letterate, Venezia, Fondazione Giorgio Cini, 2004.

Serafin, S., "Syria Poletti: la scrittura della marginalità", Oltreoceano. Vol. 2, 2008. pp.145-155.

Sodini, E., "Il fondo Bevilacqua: un itinerario tra famiglia, patriottismo femminile ed emancipazione", Scritture femminili e storia. Laura Guidi ed., Napoli, ClioPress. 2004, pp. 331-350.

Tomeucci, L., Messina nel Risorgimento. Contributo agli studi sull'unità d'Italia, Milano, Giuffrè Editore, 1963.
Verdirame, R., Narratrici e lettrici (1850-1950). Le letture della nonna dalla contessa Lara a Luciana Peverelli, Padova, libreriauniversitaria.it, 2009. 